Archive for

Organic Chemistry
Arkivoc 2017, part iii, 217-224

\title{
Enantioselective synthesis of a substituted cyclopentanone with all-carbon quaternary stereocenter
}

Antonina V. Lozanova, Andrei V. Stepanov, Mikhail V. Zlokazov, and Vladimir V. Veselovsky*

N. D. Zelinsky Institute of Organic Chemistry, Russian Academy of Sciences, 47 Leninsky prosp., 119991 Moscow, Russian Federation

Email:ves@ioc.ac.ru

\section{Dedicated to Prof. Oleg Rakitin on the occasion of his $65^{\text {th }}$ birthday}

Received 05-17-2017

Accepted 07-01-2017

Published on line $07-23-2017$

\section{Abstract}

The enantioselective synthesis of a substituted cyclopentanone with an all-carbon quaternary stereocenter was performed using an asymmetric nitroaldol condensation involving metal-complex catalysis and stereocontrolled silyl nitronate intramolecular [3+2] cycloaddition reaction in the key steps of the synthesis.

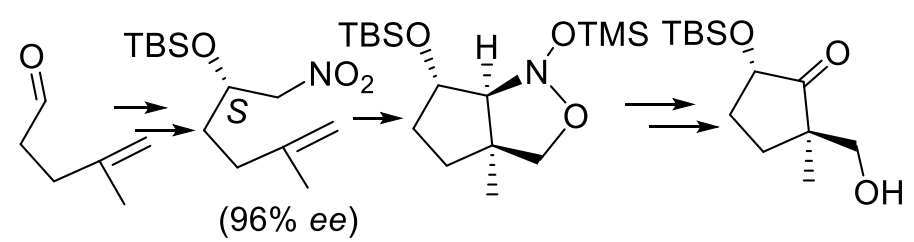

Keywords: Enantioselective synthesis; substituted cyclopentanone; silyl nitronate; intramolecular cycloaddition 


\section{Introduction}

Enantioselective construction of all-carbon quaternary stereocenters, i.e. centers in which the carbon atom bears four different carbon substituents, remains one of the most challenging subjects in modern synthetic chemistry because creation of such centers is complicated by steric repulsion between the carbon substituents. ${ }^{1}$ However there is a strong demand for the development of effective methods for the asymmetric synthesis of organic compounds containing such structural units which were revealed in a variety of natural compounds and bioactive molecules. In particular, many terpenoids, ${ }^{1-5}$ alkaloids ${ }^{1,6,7}$ and prostaglandin analogs ${ }^{8}$ contain substituted cyclopentane fragments containing such chiral centers.

Currently, there are few direct C-C bond formation reactions that have been successfully applied for the construction of all-carbon quaternary stereocenters. ${ }^{1,9,10}$ Among them the enantioselective catalysis for allylic alkylation, ${ }^{1,9-13}$ conjugation addition, ${ }^{14,15}$ Diels-Alder reactions ${ }^{16}$ and some other reactions have been used..$^{17,18}$ There are significantly fewer examples of efficient control of the stereogenic process and stereochemistry of the newly formed quaternary center directed by chiral center or centers present initially in the starting materials. ${ }^{19-21}$ Nevertheless, using the substrate-controlled stereo-induction of an easily accessible stereogenic center is a very attractive strategy to create another one that is more difficult to establish by conventional methods. We surmised that the advantages of this approach could additionally be shown using an annulation reaction by intramolecular dipolar [3+2] cycloaddition of silyl nitronates generated from chiral unsaturated nitro compounds. Previously we have demonstrated 22,23 a strong stereocontrol of the formation of substituted cyclopentane and cyclohexane rings in such cycloadditions directed by a substituent on the corresponding nitronate derived from the starting nitroolefin (cf. refs 24,25) (Scheme 1).

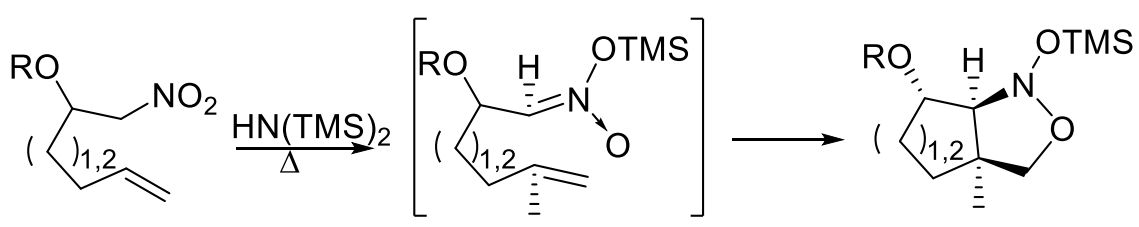

\section{Scheme 1}

Herein we describe an application of the intramolecular silyl nitronate cycloaddition reaction in the synthesis of a highly enantioenriched ( 96 \% ee) substituted cyclopentanone with an all-carbon quaternary stereocenter in the molecule.

\section{Results and Discussion}

The key unsaturated nitroalcohol 1 (96\% ee, HPLC data) in the synthesis of the target compound was obtained by enantioselective Henry reaction of 4-methylpentenal 2 with nitromethane promoted by a catalytic system proposed earlier ${ }^{26}$ for a similar process (Scheme 2). The absolute configuration of the newly obtained compound 1 was assigned by analogy with that of its homologue synthesized under the same reaction conditions. ${ }^{26}$ For further measurement of the enantiomeric excess by chiral HPLC, racemic nitroalcohol ( \pm )-1 was obtained by a convenient procedure. 


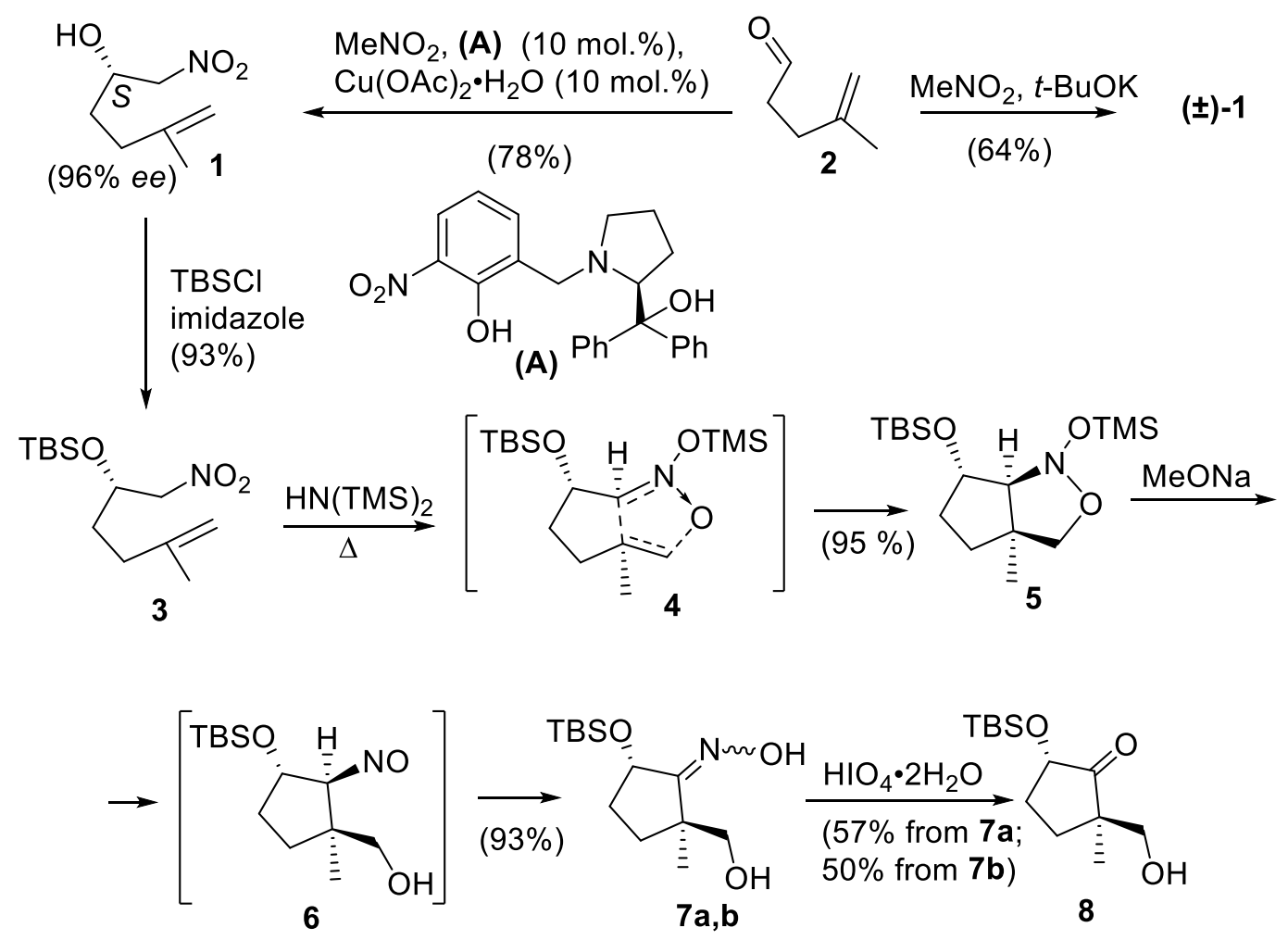

\section{Scheme 2}

The nitroalcohol 1 thus obtained was converted into silyl ether 3 . Heating the latter with 1,1,1,3,3,3hexamethyldisilazane at $115^{\circ} \mathrm{C}$ yields (via formation of nitronate 4) a rather labile cyclopentaisoxazolidine 5. The intramolecular cycloaddition proceeds highly stereoselectively with formation of the sole stereoisomer with trans-disposition of the OTBS substituent relative to the annulated isoxazolidine ring (cf. refs. 22-25). Further transformation of cyclopentaisoxazolidine $\mathbf{5}$ consisted in isoxazolidine fragment opening upon the action of sodium methoxide as described earlier for related compounds (cf. ref. 23). In this case it gives a mixture of isomeric oximes $7 \mathbf{a}, \mathbf{b}$ (anti-/syn- $\approx 12: 1,{ }^{1} \mathrm{H}$ NMR data) through initial formation of plausible nitroso intermediate $\mathbf{6}$. The configuration of the $\mathrm{C}=\mathrm{N}$ double bond in isomers $\mathbf{7 a}$ and $\mathbf{7 b}$ was established from ${ }^{13} \mathrm{C}$ NMR spectroscopic data, because it is known ${ }^{27,28}$ that the oxime hydroxyl group exerts a substantial shielding effect on the adjacent $\alpha$-carbon atom.

Deoximination of oximes $7 \mathbf{a}$ and $7 \mathbf{b}$ was carried out in the absence of a solvent by triturating with $\mathrm{HIO}_{4} \cdot 2 \mathrm{H}_{2} \mathrm{O}$ in a mortar for a short time ( $c f$. ref. 29) to give desired cycloalkanone 8 in moderate yields in both cases. Enantiomeric excess of ketone $\mathbf{8}$ is apparently not less than $96 \%$ ee since chiral center which was initially in the starting nitroalcohol 1 and the newly induced quaternary stereocenter was not affected during the subsequent stages of the synthesis.

The relative configuration of cyclopentanone 8 substituents was additionally confirmed by $1 D$ NOE experiment. Methyl group at $\mathrm{C}(2)$ shows considerable NOE with $\mathrm{H}-4 \alpha$-hydrogen (Fig. 1). Its trans-orientation to $\mathrm{H}-5$ was established by comparison of coupling constants between hydrogens $\mathrm{H}-4$ and $\mathrm{H}-5$ and these constants values calculated by DFT method using B3LYP functional and aug-cc-pvtz basis set. 


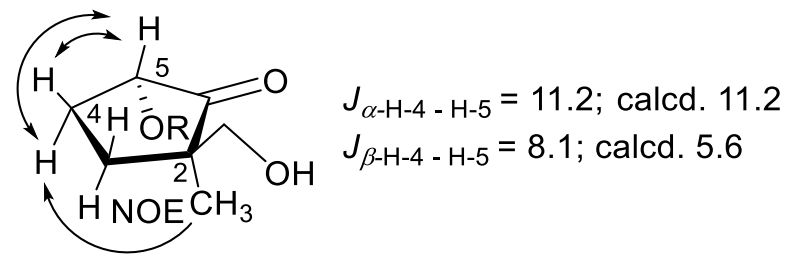

Figure 1. Structure of cyclopentanone (8).

\section{Conclusions}

In summary, we have developed a highly diastereoselective and enantioselective synthesis of a substituted cyclopentanone containing an all-carbon quaternary stereocenter. To the best of our knowledge, this is the first example of the construction of a cyclopentane core with such a structural feature using an intramolecular [3+2] cycloaddition reaction of silyl nitronate generated from an available enantioenriched nitro compound. We believe this methodology has potential to be utilized for the synthesis a number of natural cyclopentanoids.

\section{Experimental Section}

General. All reactions involving moisture-sensitive chemicals were carried out under positive pressure of argon with magnetic stirring. Commercially available chemicals were used without further purification. Solvents, including petroleum ether with bp 40-70 ${ }^{\circ} \mathrm{C}$, were purified and dried using standard procedures. Starting 4-methylpent-4-enal was prepared according to known procedure. ${ }^{30}$ Melting points were measured with a Kofler hot-stage apparatus. HRMS spectra (ESI) were obtained with a Bruker micrOTOF II mass spectrometer. IR spectra were recorded with Bruker ALPHA-T spectrometer. Optical rotation values were measured on a Jasco P-2000 polarimeter. ${ }^{1} \mathrm{H}$ and ${ }^{13} \mathrm{C} N M R$ spectra were recorded in $\mathrm{CDCl}_{3}$ or $\mathrm{C}_{6} \mathrm{D}_{6}$ at $303 \mathrm{~K}$ on a Bruker AM-300 spectrometer. Chemical shifts were reported relative to $\mathrm{CHCl}_{3}\left(\delta_{\mathrm{H}}=7.27\right.$ and $\left.\delta_{\mathrm{C}}=77.0\right)$ or $\mathrm{C}_{6} \mathrm{H}_{6}$ $\left(\delta_{H}=7.17\right.$ and $\left.\delta_{C}=128.6\right)$. TLC was performed on silica-coated glass plates (Merck, silica gel 60 F254). Visualization was accomplished by treating the plates with $\mathrm{KMnO}_{4}\left(0.5 \%\right.$ in $\left.\mathrm{H}_{2} \mathrm{O}\right)$. Flash chromatography was performed on silica gel 60 (Merck, 230-400 mesh) with the indicated eluents. For analytical HPLC a chiral phase Kromasil ${ }^{\circledR} 3$ CelluCoat (column $4.6 \times 150 \mathrm{~mm}$ ) was used. Sonication was performed with an ultrasonic bath UZV-1/100-TN at $44 \mathrm{kHz}(75 \mathrm{~W})$.

(2S)-5-Methyl-1-nitrohex-5-en-2-ol (1). A suspension of ligand (A) $(0.61 \mathrm{~g}, 1.51 \mathrm{mmol})$ and $\mathrm{Cu}(\mathrm{OAc})_{2} \cdot \mathrm{H}_{2} \mathrm{O}(0.33$ g, $1.65 \mathrm{mmol}$ ) in anhydrous $i-\mathrm{PrOH}(50 \mathrm{~mL})$ was ultrasonicated for $10 \mathrm{~min}$ at $20^{\circ} \mathrm{C}$, then aldehyde 2 (2.94 g, $29.96 \mathrm{mmol})$ and $\mathrm{MeNO}_{2}(16 \mathrm{ml})$ were added to the formed solution. The reaction mixture was kept for $40 \mathrm{~h}$ at $20{ }^{\circ} \mathrm{C}$ until complete consumption of the starting aldehyde (TLC monitoring), and concentrated under reduced pressure. Purification of the residue by column chromatography over silica gel (elution with petroleum ether/t-BuOMe, 3:1) afforded nitro alcohol 1 in the yield of $3.72 \mathrm{~g}(78 \%)$, oil, $R_{f} 0.23$ (petroleum ether/tBuOMe, 3:1), bp $65-68{ }^{\circ} \mathrm{C}$ (0.08 Torr), $[\alpha]_{\mathrm{D}}^{20}+5.8$ (c 1.00, $\mathrm{CH}_{2} \mathrm{Cl}_{2}$ ). IR (neat): 3425, 2969-2858, 1650, 1556, 1447, 1423, 1383, 1206, 1100, $891 \mathrm{~cm}^{-1} .{ }^{1} \mathrm{H}$ NMR (300 MHz, CDCl $): \delta 4.77(\mathrm{~s}, 1 \mathrm{H}, \mathrm{H}-6), 4.71\left(\mathrm{~s}, 1 \mathrm{H}, \mathrm{H}^{\prime}-6\right), 4.26-4.51$ $(\mathrm{m}, 3 \mathrm{H}, 2 \times \mathrm{H}-1, \mathrm{H}-2), 2.88$ (br. s, $1 \mathrm{H}, \mathrm{OH}), 2.17(\mathrm{~m}, 2 \mathrm{H}, \mathrm{H}-4), 1.74\left(\mathrm{~s}, 3 \mathrm{H}, \mathrm{CH}_{3}\right), 1.66(\mathrm{~m}, 2 \mathrm{H}, \mathrm{H}-3) .{ }^{13} \mathrm{C} \mathrm{NMR}(75$ 
$\mathrm{MHz}_{\mathrm{CDCl}}$ ): $\delta 141.40$ (C-5), 110.99 (C-6), 80.59 (C-1), 68.34 (C-2), 33.27 (C-4), 31.44 (C-3), 22.25 (CH $) . \mathrm{HRMS}$ (ESI) calcd. for $\mathrm{C}_{7} \mathrm{H}_{13} \mathrm{NO}_{2}[\mathrm{M}+\mathrm{H}]^{+}:$160.0968, found 160.0972. HPLC: hexane/i-PrOH, 98/2, flow rate 1.5 $\mathrm{mL} / \mathrm{min}, 30^{\circ} \mathrm{C}, \lambda=210 \mathrm{~nm}, t_{\mathrm{r}}=9.3$ (minor); $t_{\mathrm{r}}=9.7 \mathrm{~min}$ (major); calcd. $96 \%$ ee.

( \pm )-5-Methyl-1-nitrohex-5-en-2-ol (( \pm )-1). To a solution of aldehyde 2 (1.27 g, $12.94 \mathrm{mmol})$ and $\mathrm{MeNO}_{2}(1.63 \mathrm{~g}$, $26.77 \mathrm{mmol})$ in a mixture of $t-\mathrm{BuOH}$ and $\operatorname{THF}(1: 1,7 \mathrm{ml})$, stirred under argon at $20^{\circ} \mathrm{C}, t-\mathrm{BuOK}(0.14 \mathrm{~g}, 1.25$ $\mathrm{mmol}$ ) was added in a small portions. The reaction mixture was stirred at $20{ }^{\circ} \mathrm{C}$ for $1.5 \mathrm{~h}$ and then diluted with water and extracted with $t-\mathrm{MeOBu}$. The extract was washed with water and brine, dried with $\mathrm{Na}_{2} \mathrm{SO}_{4}, a$ and concentrated under reduced pressure. Purification of the residue by column chromatography over silica gel (gradient elution with petroleum ether $\rightarrow$ petroleum ether/t-BuOMe, 7:3) afforded 1.31 г (64 \%) nitroalcohol ( \pm )-1 as a colorless liquid, bp69-72 ${ }^{\circ} \mathrm{C}$ (0.09 Torr), physical characteristics of which (boiling point, NMR spectra and retention times of enantiomers) were almost the same as above described for the optically active product.

(5S)-5-t-Butyl(dimethyl)silyloxy-2-methyl-6-nitrohex-1-ene (3). Imidazole (2.86 g, $42.0 \mathrm{mmol}$ ) was added to a stirred solution of nitro alcohol 1 (3.18 g, $19.98 \mathrm{mmol})$ and TBSCl $(3.16 \mathrm{~g}, 20.97 \mathrm{mmol})$ in DMF (15 mL) under argon at $20^{\circ} \mathrm{C}$. The reaction mixture was stirred at $20^{\circ} \mathrm{C}$ for $15 \mathrm{~h}$ and then diluted with water and extracted with $t$-MeOBu . The extract was washed with water and brine, dried with $\mathrm{Na}_{2} \mathrm{SO}_{4}$, and concentrated under reduced pressure. Purification of the residue by column chromatography over silica gel (gradient elution with petroleum ether $\rightarrow$ petroleum ether/t-BuOMe, 9:1) afforded silyl ether 3 in the yield of $5.08 \mathrm{~g}(93 \%)$, oil, $R_{f}$ 0.52 (petroleum ether/t-BuOMe, 9:1), bp 78-80 ${ }^{\circ} \mathrm{C}$ (0.08 Torr), $[\alpha]_{\mathrm{D}}^{28}+26.9$ (c 1.00, $\mathrm{CH}_{2} \mathrm{Cl}_{2}$ ). IR (neat): 29552859, 1650, 1558, 1473, 1386, 1258, 1115, 981, 838, 811, $779 \mathrm{~cm}^{-1} .{ }^{1} \mathrm{H} \mathrm{NMR}\left(300 \mathrm{MHz}, \mathrm{CDCl}_{3}\right): \delta 4.77(\mathrm{~s}, 1 \mathrm{H}$, $\mathrm{H}-1), 4.71\left(\mathrm{~s}, 1 \mathrm{H}, \mathrm{H}^{\prime}-1\right), 4.41(\mathrm{~m}, 3 \mathrm{H}, \mathrm{H}-5, \mathrm{H}-6), 2.07(\mathrm{~m}, 2 \mathrm{H}, \mathrm{H}-3), 1.74\left(\mathrm{~s}, 3 \mathrm{H}, \mathrm{CH}_{3}\right), 1.71(\mathrm{~m}, 2 \mathrm{H}, \mathrm{H}-4), 0.87(\mathrm{~s}, 9$ $\mathrm{H}, \mathrm{CH}_{3} \mathrm{CSi}$ ), 0.09, $0.04\left(\mathrm{~s}, 6 \mathrm{H}, \mathrm{CH}_{3} \mathrm{Si}\right) .{ }^{13} \mathrm{C} \mathrm{NMR}\left(75 \mathrm{MHz}_{2} \mathrm{CDCl}_{3}\right): \delta=144.40$ (C-2), 110.67 (C-1), 80.95 (C-6), 69.74 (C-5), 33.09 (C-3), 32.60 (C-4), $25.61\left(\mathrm{CH}_{3} \mathrm{CSi}\right) 22.25\left(\mathrm{CH}_{3}\right), 17.90\left(\mathrm{CH}_{3} \mathrm{CSi}\right),-4.68,-5.19$ (CH $\left.\mathrm{CH}\right) . \mathrm{HRMS}$ (ESI) calcd. for $\mathrm{C}_{13} \mathrm{H}_{27} \mathrm{NO}_{3} \mathrm{Si}[\mathrm{M}+\mathrm{H}]^{+}:$:274.1833; found 274.1839 .

(3aS,6S,6aS)-6-t-Butyl(dimethyl)silyloxy-3a-methyl-1-(trimethylsilyloxy)perhydrocyclopenta[c]isoxazole (5). A stirred under argon mixture of compound 3 (3.82 g, $13.97 \mathrm{mmol}), \mathrm{Et}_{3} \mathrm{~N}(1.67 \mathrm{~g}, 16.50 \mathrm{mmol}), \mathrm{HMPA}$ (0.5 g, $2.79 \mathrm{mmol}$ ) and HMDS (18 mL), was heated at $115^{\circ} \mathrm{C}$ for $10 \mathrm{~h}$ and then concentrated under reduced pressure. The residue was distilled off. Compound 5 was obtained in a yield of $4.58 \mathrm{~g}$ (95\%) as a colorless oil with bp $85-$ $89{ }^{\circ} \mathrm{C}\left(0.08\right.$ Torr). IR (neat): 2957-2858, 1462, 1251, 1072, 921, 881, 841, $776 \mathrm{~cm}^{-1} .{ }^{1} \mathrm{H} \mathrm{NMR}(300 \mathrm{MHz}, \mathrm{CDCl} 3): \delta$ 4.10 (d, $1 \mathrm{H}, \mathrm{H}-3, J=8.0$ ), 4.03 (m, $1 \mathrm{H}, \mathrm{H}-6), 3.95$ (d, $\left.1 \mathrm{H}, \mathrm{H}^{\prime}-3, J=8.0\right), 3.69$ (d, $\left.1 \mathrm{H}, \mathrm{H}-6 \mathrm{a}, J=7.9\right), 1.49-2.03$ (m, $4 \mathrm{H}, \mathrm{H}-4, \mathrm{H}-5), 1.16\left(\mathrm{~s}, 3 \mathrm{H}, \mathrm{CH}_{3}\right), 0.87\left(\mathrm{~s}, 9 \mathrm{H}, \mathrm{CH}_{3} \mathrm{CSi}\right), 0.12,0.08$ and $0.04\left(\mathrm{~s}, 15 \mathrm{H}, \mathrm{CH}_{3} \mathrm{Si}\right)$. HRMS (ESI) calcd. for $\mathrm{C}_{16} \mathrm{H}_{35} \mathrm{NO}_{3} \mathrm{Si}_{2}[\mathrm{M}+\mathrm{H}]^{+}:$346.2228; found 346.2229.

(1E,2S,5S)-5-t-Butyl(dimethyl)silyloxy-2-(hydroxymethyl)-1-(hydroxyimino)-2-methylcyclopentane (7a) and (1Z,2S,5S)-5-t-butyl(dimethyl)silyloxy-2-(hydroxymethyl)-1-(hydroxyimino)-2-methylcyclopentane (7b). To a stirred under argon suspension of MeONa $(1.24 \mathrm{~g}, 23.04 \mathrm{mmol})$ in $\mathrm{PhH}(25 \mathrm{~mL})$, a solution of isoxazolidine 5 $(4.48 \mathrm{~g}, 13.0 \mathrm{mmol})$ in $\mathrm{PhH}(10 \mathrm{~mL})$ was added dropwise at $20^{\circ} \mathrm{C}$. The reaction mixtur was stirred at $20^{\circ} \mathrm{C}$ for 2 $\mathrm{h}$ and neutralized with $10 \%$ aqueous $\mathrm{HCl}$. The aqueous layer was separated and extracted with EtOAc. Combined organic layers were washed with water and brine dried with $\mathrm{NaSO}_{4}$, and the solvent was removed under reduced pressure. Column chromatography of the residue over silica gel (elution with EtOAc) afforded oximes $7 \mathrm{a}$ and $7 \mathrm{~b}$. Oxime $7 \mathrm{a}$ (yield $3.05 \mathrm{~g}, 86 \%$ ), colorless crystals, $\mathrm{mp} 76-7{ }^{\circ} \mathrm{C}$ (from petroleum ether), $[\alpha]_{\mathrm{D}}^{28}$ +110.6 (c 1.00, $\left.\mathrm{CH}_{3} \mathrm{OH}\right)$. IR (KBr): 3331, 2954-2859, 1472-1346, 1254, 1132, 1078, 1032, 943, 836, $776 \mathrm{~cm}^{-1} .{ }^{1} \mathrm{H}$ NMR (300 MHz, C6 $\left.\mathrm{D}_{6}\right)$ : $\delta 9.65$ (br.s, $\left.1 \mathrm{H}, \mathrm{NOH}\right), 5.05$ (t, $1 \mathrm{H}, \mathrm{H}-5, \mathrm{~J}=3.8$ ), 3.70 (br.s $\left.1 \mathrm{H}, \mathrm{OH}\right), 3.62(\mathrm{~d}, 1 \mathrm{H}, \mathrm{HCO}, J$ $=10.1), 3.55\left(\mathrm{~d}, 1 \mathrm{H}, \mathrm{H}^{\prime} \mathrm{CO}, J=10.1\right), 1.51-1.81(\mathrm{~m}, 4 \mathrm{H}, \mathrm{H}-3, \mathrm{H}-4), 1.43\left(\mathrm{~s}, 3 \mathrm{H}, \mathrm{CH}_{3}\right), 0.99\left(\mathrm{~s}, 9 \mathrm{H}, \mathrm{CH}_{3} \mathrm{CSi}\right), 0.28$, $0.33\left(\mathrm{~s}, 6 \mathrm{H}, \mathrm{CH}_{3} \mathrm{Si}\right) .{ }^{13} \mathrm{C} \mathrm{NMR}\left(75 \mathrm{MHz}, \mathrm{C}_{6} \mathrm{D}_{6}\right): \delta=170.17(\mathrm{C}=\mathrm{NOH}), 69.87\left(\mathrm{CH}_{2} \mathrm{O}\right), 69.51(\mathrm{C}-5), 46.39(\mathrm{C}-2), 33.68$, 
33.72 (C-3, C-4), $26.67\left(\mathrm{CH}_{3}-\mathrm{CSi}\right), 24.75\left(\mathrm{CH}_{3}-2\right), 19.00\left(\mathrm{CH}_{3} \mathrm{CSi}\right),-4.04,-4.31\left(\mathrm{CH}_{3} \mathrm{Si}\right) . \mathrm{HRMS}$ (ESI) calcd. for $\mathrm{C}_{13} \mathrm{H}_{27} \mathrm{NO}_{3} \mathrm{Si}[\mathrm{M}+\mathrm{H}]^{+}: 274.1833$ and $[\mathrm{M}+\mathrm{Na}]^{+}: 296.1652$; found 274.1837 and 296.1655. Oxime $7 \mathrm{~b}$ (yield 0.25 g, 7\%), colorless crystals, m.p. $57-58{ }^{\circ} \mathrm{C}$ (from petroleum ether), $[\alpha]_{\mathrm{D}}^{28}+32.7$ (c 1.00, $\left.\mathrm{CH}_{3} \mathrm{OH}\right) . \mathrm{IR}(\mathrm{KBr}): 3264$, 2858-2956, 1362-1472, 1253, 1138, 1091, 1045, 992, 937, 839, 781, $670 \mathrm{~cm}^{-1}$. ${ }^{1} \mathrm{H} N M R\left(300 \mathrm{MHz}, \mathrm{C}_{6} \mathrm{D}_{6}\right): \delta=$ 9.65 (br.s, $1 \mathrm{H}, \mathrm{NOH}), 4.54$ (t, $1 \mathrm{H}, \mathrm{H}-5, J=6.6), 4.09$ (d, $1 \mathrm{H}, \mathrm{HCO}, J=10.7), 3.58\left(\mathrm{~d}, 1 \mathrm{H}, \mathrm{H}^{\prime} \mathrm{CO}, J=10.7\right), 3.18$ (br.s $1 \mathrm{H}, \mathrm{OH}), 1.64-1.95(\mathrm{~m}, 4 \mathrm{H}, \mathrm{H}-3, \mathrm{H}-4), 1.41\left(\mathrm{~s}, 3 \mathrm{H}, \mathrm{CH}_{3}\right), 0.99\left(\mathrm{~s}, 9 \mathrm{H}, \mathrm{CH}_{3} \mathrm{CSi}\right), 0.33,0.29\left(\mathrm{~s}, 6 \mathrm{H}, \mathrm{CH}_{3} \mathrm{Si}\right)$. ${ }^{13} \mathrm{C}$ NMR $\left(75 \mathrm{MHz}, \mathrm{C}_{6} \mathrm{D}_{6}\right): \delta=167.82(\mathrm{C}=\mathrm{NOH}), 76.06$ (C-5), $68.66\left(\mathrm{CH}_{2} \mathrm{O}\right), 48.73(\mathrm{C}-2), 34.11,33.43(\mathrm{C}-3, \mathrm{C}-4)$, $26.74\left(\mathrm{CH}_{3} \mathrm{CSi}\right), 20.61\left(\mathrm{CH}_{3}-2\right), 19.03\left(\mathrm{CH}_{3} \mathrm{CSi}\right),-3.99,-3.73\left(\mathrm{CH}_{3} \mathrm{Si}\right) . \mathrm{HRMS}(\mathrm{ESI})$ calcd. for $\mathrm{C}_{13} \mathrm{H}_{27} \mathrm{NO}_{3} \mathrm{Si}[\mathrm{M}+\mathrm{H}]^{+}$: 274.1833 and $[\mathrm{M}+\mathrm{Na}]^{+}:$:296.1652; found 274.1821 and 296.1647.

(2R,5S)-5-t-Butyl(dimethyl)silyloxy-2-hydroxymethyl-2-methylcyclopentan-1-one (8). A: Oxime $7 \mathrm{a}$ (0.60 g, $2.19 \mathrm{mmol})$ and $\mathrm{HIO}_{4} \cdot 2 \mathrm{H}_{2} \mathrm{O}(0.25 \mathrm{~g}, 1.09 \mathrm{mmol})$ was thoroughly mixed and ground in an agate mortar for 2 min. The resulting homogeneous oily material was extracted with $t$-BuOMe. The extract was washed with solution of $\mathrm{Na}_{2} \mathrm{~S}_{2} \mathrm{O}_{3}$, water and brine dried with $\mathrm{Na}_{2} \mathrm{SO}_{4}$, and the solvent was removed under reduced pressure. Purification of the residue by column chromatography over silica gel (gradient elution with $\mathrm{CH}_{2} \mathrm{Cl}_{2} \rightarrow \mathrm{CH}_{2} \mathrm{Cl}_{2} / t$ BuOMe, 9:1) afforded cyclopentanone 8 in the yield of $0.32 \mathrm{~g}\left(57 \%\right.$ ), colorless crystals, $\mathrm{mp} 46-47{ }^{\circ} \mathrm{C}$ (from petroleum ether), $[\alpha]_{\mathrm{D}}^{28}+75.1$ (c 1.00, $\mathrm{CH}_{2} \mathrm{Cl}_{2}$ ). IR (KBr): 3468, 2967-2858, 1751, 1463, 1362, 1254, 1173, 1147, 1054, 995, 839, 780. ${ }^{1} \mathrm{H}$ NMR (300 MHz, CDCl $)$ : $\delta=4.05$ (d.d, $1 \mathrm{H}, \mathrm{H}-5, \mathrm{~J}=10.9, \mathrm{~J}=8.2$ ), 3.65 (d, $1 \mathrm{H}, \mathrm{HCO}, \mathrm{J}=$ 10.6), 3.39 (d, $\left.1 \mathrm{H}, \mathrm{H}^{\prime} \mathrm{CO}, J=10.6\right)$, and 1.94-2.28 1.62-1.88 (both $\mathrm{m}, 4 \mathrm{H}, \mathrm{H}-3$, and $\left.\mathrm{H}-4\right), 1.03\left(\mathrm{~s}, 3 \mathrm{H}, \mathrm{CH}_{3}\right)$, $0.92\left(\mathrm{~s}, 9 \mathrm{H}, \mathrm{CH}_{3} \mathrm{CSi}\right), 0.14\left(\mathrm{~s}, 3 \mathrm{H}, \mathrm{CH}_{3} \mathrm{Si}\right), 0.09\left(\mathrm{~s}, 3 \mathrm{H}, \mathrm{CH}_{3} \mathrm{Si}\right) .{ }^{13} \mathrm{C} \mathrm{NMR}\left(75 \mathrm{MHz} \mathrm{CDCl}_{3}\right): \delta=220.30$ (C=O), 77.10 (C-2), $67.60\left(\mathrm{CH}_{2} \mathrm{O}\right), 48.13$ (C-5), 28.68, 26.97, (C-3, C-4), $25.81\left(\mathrm{CH}_{3} \mathrm{CSi}\right), 20.26\left(\mathrm{CH}_{3}-5\right), 18.43\left(\mathrm{CH}_{3} \mathrm{CSi}\right),-4.51$, -4.99, $\left(\mathrm{CH}_{3} \mathrm{Si}\right)$. HRMS (ESI) calcd. for $\mathrm{C}_{13} \mathrm{H}_{26} \mathrm{O}_{3} \mathrm{Si}\left[\mathrm{M}+\mathrm{H}^{+}: 259.1724\right.$ and $[\mathrm{M}+\mathrm{Na}]^{+}:$281.1543; found 259.1721 and 281.1535. B: The sample of cyclopentanone 8 with almost the same characteristics (m.p., optical rotation and NMR spectra) was prepared by similar procedure from oxime $\mathbf{7 b}$ in the yield $50 \%$.

\section{Acknowledgements}

This work was supported by the Russian Foundation for Basic Research (Project No. 13-03-00197).

\section{References}

1. Quaternary Stereocenters: Challenges and Solutions for Organic Synthesis; Christoffers J.; Baro A., Eds.; Wiley-VCH: Weinheim. 2005, 336 pp.

2. Abbas, H. K.; Mirocha, C. J. Appl. Environ. Microbiol. 1988, 54, 1268.

3. Wellington, K. D.; Cambie, R. C.; Rutledge, P. S.; Bergquist, P. R. J. Nat. Prod. 2000, 63, 79. http://dx.doi.org/10.1021/np9903494

4. Asai, R.; Mitsuhashi, S.; Shigetomi, K.; Miyamoto, T.; Ubukata, M. J. Antibiot. 2011, 64, 693. http://dx.doi.org/10.1038/ja.2011.73

5. Bîschleb M.; Dorich, S.; Hanessian, S.; Tao, D.; Schenthal, K. B.; Overman, L. E. Angew. Chem. Int. Ed. 2016, $55,4156$.

\section{http://dx.doi.org/10.1002/anie.201507549}

6. Oh, H.; Swenson, D. C.; Gloer, J. B.; Wicklow, D. T.; Dowd, P. F. Tetrahedron Lett. 1998, 39, 7633. http://dx.doi.org/10.1016/S0040-4039(98)01692-X 
7. Takayama, H.; Katakawa, K.; Kitajima, M.; Yamaguchi, K.; Aimi, N. Tetrahedron Lett. 2002, $43,8307$. http://dx.doi.org/10.1016/S0040-4039(02)02026-9

8. Nakamura, N.; Sakai, K. Tetrahedron Lett. 1976, 17, 2049. http://dx.doi.org/10.1016/S0040-4039(00)93814-0

9. Trost, B. M.; Jiang, C. Synthesis 2006, 369. http://dx.doi.org/10.1055/s-2006-926302

10. Das, J. P.; Marek, I. Chem. Commun. 2011, 47, 4593.

http://dx.doi.org/10.1039/C0CC05222A

11. Mohr, J. T.; Stoltz, B. M. Chem. Asian J. 2007, 2, 1476.

http://dx.doi.org/10.1002/asia.200700183

12. Hong, A. Y.; Stoltz, B. M. Eur. J. Org. Chem. 2013, 2745.

http://dx.doi.org/10.1002/ejoc.201201761

13. Craig, R. A. II; Loskot, S. A.; Mohr, J. T.; Behenna, D. C.; Harned, A. M.; Stoltz, B. M. Org. Lett. 2015, 17, 5160.

http://dx.doi.org/10.1021/acs.orglett.5b02376

14. Shintani, R.; Takeda, M.; Nishimura, T.; Hayashi, T. Angew. Chem. Int. Ed. 2010, 49, 3969.

http://dx.doi.org/10.1002/anie.201000467

15. Kikushima, K.; Holder, J. C.; Gatti, M.; Stoltz, B. M. J. Am. Chem. Soc. 2011, 133, 6902.

http://dx.doi.org/10.1021/ja200664x

16. Corey, E. J. Angew. Chem. Int. Ed. 2002, 41, 1650.

http://dx.doi.org/10.1002/1521-3773(20020517)41:10<1650::AID-ANIE1650>3.0.CO;2-B

17. Zhou, F.; Cheng, G.-J.; Yang, W.; Long, Y.; Zhang, S.; Wu, Y.-D.; Zhang, X.; Cai, Q. Angew. Chem. Int. Ed. 2014, 53, 9555.

http://dx.doi.org/10.1002/anie.201405575

18. Zhu, D.-Y.; Xu, M.-H.; Tu, Y.-Q.; Zhang, F.-M.; Wang, S.-H. Chem. Eur. J. 2015, 21, 15502.

http://dx.doi.org/10.1002/chem.201502700

19. Takao, K.-I; Sakamoto, S.; Touati, M. A.; Kusakawa, Y.; Tadano, K.-I. Molecules. 2012, 17, 13330.

http://dx.doi.org/10.3390/molecules171113330

20. Fujii, T.; Orimoto, K.; Nakada, M. Tetrahedron Lett. 2014, 55, 1100.

http://dx.doi.org/10.1016/j.tetlet.2013.12.110

21. Penrose, S. D.; Stott, A. J.; Breccia, P.; Haughan, A. F.; Bürli, R W.; Jarvis, R. E.; Dominguez, C. Org. Lett. 2015, 17, 1401.

http://dx.doi.org/10.1021/acs.orglett.5b00207

22. Lozanova, A.V.; Chizhov, A.O.; Veselovsky, V.V. Russ. Chem. Bull. 2011, 60, 2331.

http://dx.doi.org/10.1007/s11172-011-0357-7

23. Lozanova, A.V.; Zlokazov, M.V.; Veselovsky, V.V. Russ. Chem. Bull. 2014, 63, 696.

http://dx.doi.org/10.1007/s11172-014-0494-x

24. Roger, P.-Y.; Durand, A.-C.; Rodriguez, J.; Dulcère, J.-P. Org. Lett. 2004, 6, 2027.

http://dx.doi.org/10.1021/ol049394f

25. Ioffe, S. L. In: Nitrile Oxides, Nitrones, and Nitronates in Organic Synthesis. Novel Strategies in Synthesis; Feuer H. Ed.; John Wiley: New Jersey, 2008; p 558.

26. Veselovsky, V. V.; Stepanov, A. V. Russ. Chem. Bull. 2014, 63, 422.

http://dx.doi.org/10.1007/s11172-014-0447-4

27. Bunnell, C. A.; Fuchs, P. L. J. Org. Chem. 1977, 42, 2614. 
http://dx.doi.org/10.1021/jo00435a016

28. Faisal, S.; Basha, A. F.; Siddiqui, H.; Basha, F. Z. Synth. Commun. 2010, 40, 3101. http://dx.doi.org/10.1080/00397910903370691

29. Lozanova, A.V.; Shashkov, A.S.; Veselovsky, V.V. Russ. Chem. Bull. 2015, 64, 379. http://dx.doi.org/10.1007/s11172-015-0872-z

30. Baker, R.; Brimble, M. A. J. Chem. Soc. Perkin Trans. I. 1988, 125.

http://dx.doi.org/10.1039/P19880000125 